\title{
OPTIMAL TRACK FUSION USING BAYES FACTORS
}

\author{
Daniel McMichael, Mehmet Karan \\ The Cooperative Centre for Sensor \\ Signal and Information Processing, \\ Mawson Lakes, \\ South Australia, 5095 \\ $\{$ dwm,mehmet\}@cssip.edu.au
}

keywords: tracking, association, Bayes factor, Markov process, Kalman filter, generalized tracks.

\begin{abstract}
In deciding whether to associate and fuse a group of tracks sent from independent local trackers, use should be made of all the data supporting them during theircommon history. This paper provides a closed form expression for the relevant Bayes factor, which is the ratio of the probability that the tracks are caused by a single target to the probability that they are each caused by a different target. The expression is recursive, and it applies to processes which may include a linear Gaussian process and several discrete Markov processes. Two variants are provided, one that requires the data to be sent from local trackers to the fusion centre, and one that only requires discrete probabilities state estimates and covariance matrices.
\end{abstract}

\section{BAYES FACTORS}

The track association problem we consider concerns whether tracks estimated independently by different trackers using different data are caused by the same target. If so, the tracks should be fused. This paper provides techniques for making such decisions which use all the data from the period of their shared existence. The equations can also be formulated so that only the track estimates and covariances are needed, and the data can be thrown away. Our line of attack is to evaluate the Bayes factor [1] for the fusion hypothesis pair

$$
\begin{array}{lll}
\mathcal{H}_{0} & : & \text { Target states are equal, } \\
\mathcal{H}_{1} & : & \text { Target states are different. }
\end{array}
$$

A Bayes factor ${ }^{1}$ is the ratio of the probability of two hypotheses given relevant data $Z$. Frequently, the parameters have to be integrated out. The Bayes factor for this hypothesis pair is

$$
\frac{P\left(\mathcal{H}_{0} \mid Z\right)}{P\left(\mathcal{H}_{1} \mid Z\right)}=\frac{P\left(\mathcal{H}_{0}\right) \int p\left(Z \mid \Phi, \mathcal{H}_{0}\right) d \Phi}{P\left(\mathcal{H}_{1}\right) \int p\left(Z \mid \Theta, \mathcal{H}_{1}\right) d \Theta} .
$$

Bayes factors behave differently from generalised likelihood ratios (GLRs), in which the integrations, above, are substituted by maximisations over the parameters. GLRs may not represent the relative validity of the hypotheses because although the likelihood is maximised at the maximum likelihood estimate, the peak may be narrow, giving a small integrated likelihood $p(Z \mid \mathcal{H})$. Bayes factors do not rely on such estimates and are therefore more robust; but they are more difficult to obtain.

\section{THE TRACKING MODEL}

It is possible to treat the trackers for mixed linear Gaussian and discrete Markov processes in a unified way. Analytical association results can be found for such generalised tracks $[3,4,5]$. The joint state $X$ comprises the Gaussian kinematic state $X_{G}$ and the discrete states, $X_{D}$. We assume that the classification states and the kinematic states are independent and that the data can be partitioned into subsets $Z$ and $R ; Z$ provides information about $X_{G}$, and $R$ provides information about $X_{D}$. Under these circumstances, finite sufficient statistics exist for the states, and the posterior probabilities of $X_{G}$ and $X_{D}$ factorise:

$$
p\left(X_{G}, X_{D} \mid Z, R\right)=p\left(X_{G} \mid Z\right) P\left(X_{D} \mid R\right),
$$

and the sequential updates of the Kalman and Markov process filters are independent [4]. The value of joint tracking and classification under this model derives from the association process, which becomes more resistant to clutter and measurement noise [3].

Each sensor suite drives a local tracker. Periodically, the local trackers send information to the fusion centre (e.g. state estimates, covariance estimates and, in the case of discrete variables, the entire distribution). The track fusion and association equations we derive are applicable to both feedback and independent distributed tracking frameworks [6, p. 434 et. seq.]. In the feedback framework, the fusion centre sends fused estimates back to the local trackers. The detailed modifications needed for asynchronous updating can readily be worked out and are not described here.

Previous work by Chang, Bar-Shalom and $\mathrm{Li}[7,6]$ has provided algorithms for fusing and associating tracks of Gaussian processes using the current track state estimates and fusing fixed discrete feature states.

\footnotetext{
${ }^{1}$ The Bayes factor is the posterior odds of the two hypotheses. In the control literature it recently been referred to the marginalised likelihood ratio [2].
} 


\section{TARGET AND MEASUREMENT MODELS}

Let the Gaussian and discrete states at time $k$ be $X_{G}(k)$ and $X_{D}(k)$, respectively. The Gaussian state $X_{G}(k)$ has $n$ elements. The discrete state $X_{D}(k)$ is a compound variable ${ }^{2}$ with $C$ components: $X_{D}(k)=\left\{X_{D_{c}}(k)\right\}_{c=1}^{C}$. The component $X_{D_{c}}$, has $n_{c}$ states. The unified target state vector is therefore $X(k)=\left[X_{G}(k)^{T}, X_{D_{c}}(k), \ldots, X_{D_{C}}(k)\right]^{T}$. These components of the state vector are assumed to evolve independently of one another, and so the state transition factorises as

$$
p(X(k) \mid X(k-1))=p\left(X_{G}(k) \mid X_{G}(k-1)\right) \prod_{c=1}^{C} p\left(X_{D_{c}}(k) \mid X_{D_{c}}(k-1)\right),
$$

where the coefficients all the factors are known. The component processes evolve according to

$$
p\left(X_{G}(k) \mid X_{G}(k-1)\right)=\mathrm{N}\left[F(k-1) X_{G}(k-1), Q(k-1)\right],
$$

where $Q(k-1)$ is an appropriate positive definite matrix, and the transition probabilities,

$$
p\left(X_{D_{c}}(k) \mid X_{D_{c}}(k-1)\right) \text {, }
$$

are arbitrary. Each of the $S$ sensors provides, at each scan, an augmented measurement set $z_{s, a}(k)=\left\{z_{s}(k), r_{s, 1}(k), \ldots, r_{s, C}(k)\right\}$ to the local tracker, in which $z_{s}(k)$ is the Gaussian component, $r_{s}(k)$ is the discrete component, and $s$ is the identifier of the sensor. The measurement components are assumed to be conditionally independent between sensors and each other (e.g. all of $r_{1}(k), r_{2}(k)$ $r_{2}(k-1)$ and $z_{3}(k)$ are conditionally independent). Therefore, the likelihood of a the genereralised state vector $X(k)$ of the target, given measurement $z_{s, a}(k)$ is

$$
p\left(z_{s, a}(k) \mid X(k)\right)=p\left(z_{s}(k) \mid X_{G}(k)\right) \prod_{c=1}^{C} p\left(r_{s, c}(k) \mid X_{D_{c}}(k)\right) .
$$

The Gaussian term has mean $H_{s}(k) X_{G}(k)$ and variance $V_{s}(k)$,

$$
p\left(z_{s}(k) \mid X_{G}(k)\right)=\mathrm{N}\left[H_{s}(k) X_{G}(k), V_{s}(k)\right],
$$

and the discrete likelihoods,

$$
p\left(r_{s, c}(k) \mid x_{D_{c}}(k)\right) \quad \forall c \in\{1, \ldots, C\}, \quad \text { are arbitrary. }
$$

The set of measurements at sensor $s$ up to time $k$ is $Z_{s, a}^{k}=\left\{z_{s, a}(t)\right\}_{t=1}^{k}$. Similarly, $Z_{s}^{k}=\left\{z_{s}(t)\right\}_{t=1}^{k}$ and $R_{s, c}^{k}=\left\{r_{s, c}(t)\right\}_{t=1}^{k}$. The set of augmented measurements from all sensors up to time $k$ is $Z_{a}^{k}=\left\{Z_{s, a}^{k}\right\}_{s=1}^{S}$. Likewise, $Z^{k}=\left\{Z_{s}^{k}\right\}_{s=1}^{S}$ and $R^{k}=\left\{R_{s}^{k}\right\}_{s=1}^{S}$.

\section{DISTRIBUTED TARGET STATE INFERENCE}

In order to keep the treatment exact, we assume that the track-to-data association has already been done. In practise, any good data association algorithm can be used. The posterior distribution of the generalised track state at time $k$ is calculated recursively by Bayes rule:

$$
p\left(X(k) \mid Z_{a}^{k}\right)=\frac{p\left(Z_{a}(k) \mid X(k)\right) p\left(X(k) \mid Z_{a}^{k-1}\right)}{p\left(Z_{a}(k) \mid Z_{a}^{k-1}\right)}
$$

where $X(k)$ is the generalised state vector and $Z_{a}^{k}$ is the set of all measurements up to time $k$. The strong independence properties listed in section 3 allow the local generalised tracks to be calculated using a battery of filters, represented here as Bayes rule recursions, one for each of the $C+1$ factors of the state update equation

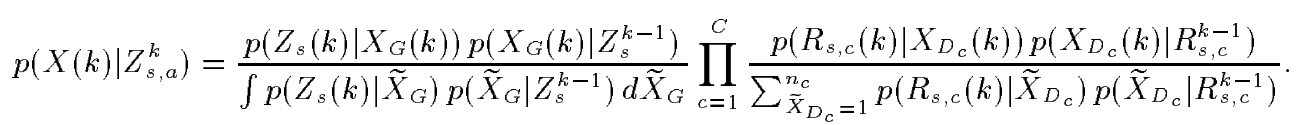

The local trackers update the Gaussian factor of their local state posterior distributions using a Kalman filter to propagate the means $\widehat{X}_{s}$ and covariance matrices $P_{s}, s \in\{1, \ldots, S\}$ (see [8], for example). Using the well-established notation, the Kalman filter update is

$$
\begin{aligned}
\widehat{X}_{s}(k \mid k) & =\widehat{X}_{s}(k \mid k-1)+P_{s}(k \mid k) H_{s}{ }^{T}(k) V_{s}(k)^{-1}\left[z_{s}(k)-H_{s} \widehat{X}_{s}(k \mid k-1)\right], \\
P_{s}(k \mid k-1) & =F(k-1) P_{s}(k-1 \mid k-1) F^{T}(k-1)+Q(k-1), \\
P_{s}^{-1}(k \mid k) & =P_{s}^{-1}(k \mid k-1)+H_{s}^{T}(k) V_{s}{ }^{-1}(k) H_{s}(k),
\end{aligned}
$$

where $V_{s}(k)$ is the covariance of the Gaussian observation at sensor $s$. The hidden Markov model inference applied here extends [9, Chap. 13]. The time and measurement updates are

$$
\begin{aligned}
p\left(X_{D_{c}}(k) \mid R_{s}^{k-1}\right) & =\sum_{j=1}^{n_{c}} p\left(X_{D_{c}}(k) \mid X_{D_{c(j)}}\right) p\left(X_{D_{c(j)}} \mid R_{s}^{k-1}\right), \\
p\left(X_{D_{c}}(k) \mid R_{s}^{k}\right) & =\frac{p\left(r_{s, c}(k) \mid X_{D_{c}}(k)\right) p\left(X_{D_{c}}(k) \mid R_{s}^{k-1}\right)}{\sum_{j=1}^{n_{c}} p\left(r_{s, c}(k) \mid X_{D_{c}(j)}\right) p\left(X_{D_{c}(j)} \mid R_{s}^{k-1}\right)} .
\end{aligned}
$$

${ }^{2}$ Although a single discrete variable can describe the state of any finite compound set of discrete variables, the number of states rapidly becomes unmanageable and it is prudent to use several components, each with few states. 


\subsection{Measurement Fusion}

In preparation for later working, we introduce the equations for fusing the plot data from several sensors directly. We consider the two sensor case; measurement fusion with multiple sensors follows the same pattern. It is assumed that sensors $i$ and $j$ obtain measurements $z_{i, a}(k)$ and $z_{j, a}(k)$ of targets with states $X_{i}(k)$ and $X_{j}(k)$, respectively. These states evolve according to (6), (7), (9) and (10). We assume there is no model mismatch. The fused tracker uses the same time updates as the local trackers, but fuses the measurements from the two sensors, resulting in the measurement probabilities

$$
p\left(z_{i}(k), z_{j}(k) \mid X_{G}(k)\right)=\mathrm{N}\left[H(k) X_{G}(k), V(k)\right],
$$

where $H(k)=\left[H_{i}^{T}(k), H_{j}^{T}(k)\right]^{T}$, and $V(k)=\left[\begin{array}{cc}V_{i}(k) & \mathbf{0} \\ \mathbf{0} & V_{j}(k)\end{array}\right]$, and

$$
p\left(r_{c}(k) \mid x_{D_{c}}(k)\right)=p\left(r_{c}^{i}(k) \mid x_{D_{c}}(k)\right) p\left(r_{c}^{j}(k) \mid x_{D_{c}}(k)\right) \quad \forall c \in\{1, \ldots, C\} .
$$

The observed distributions of the states $X_{i}(k)$ and $X_{j}(k)$ and the fused state $X(k)$ are updated according to the Kalman filter update (12), (13) and (14), and the Markov process filter update (16) and (15). Several target measurements per scan can be handled by repeating the measurement updates. If the measurements are asynchronous, the observed distribution of $X(k)$ must be updated every time a measurement arrives from either sensor.

\subsection{The integrated likelihood of the Gaussian data}

The integrated likelihood of a model $\mathcal{H}$ given a set of data $Z_{a}^{k}$ parameterised by $\Theta$ is $p\left(Z_{a}^{k} \mid \mathcal{H}\right)=\int p\left(Z_{a}^{k} \mid \mathcal{H}, \Theta\right) p(\Theta \mid \mathcal{H}) d \Theta$. Under conditional temporal independence of the data, it can be evaluated recursively:

$$
p\left(Z_{a}^{k} \mid \mathcal{H}\right)=\prod_{t=1}^{k} p\left(Z_{a}(t) \mid Z_{a}^{t-1}, \mathcal{H}\right)
$$

The independence assumptions we have made allow this probability to be factorised again:

$$
P\left(Z_{a}(k) \mid Z_{a}^{k-1}, \mathcal{H}\right)=P\left(z(k) \mid Z^{k-1}, \mathcal{H}\right) \prod_{c=1}^{C} P\left(r_{c}(k) \mid R_{c}^{k-1}, \mathcal{H}\right) .
$$

A few lines of algebra show that the recursive term for the Gaussian data is

$$
p\left(z(k) \mid Z^{k-1}, \mathcal{H}\right)=\frac{e^{-\frac{1}{2}[z(k)-H(k) \hat{X}(k \mid k-1)]^{T}\left\{H(k) P(k \mid k-1) H^{T}(k)+V(k)\right\}^{-1}[z(k)-H(k) \hat{X}(k \mid k-1)]}}{\left|2 \pi\left(H(k) P(k \mid k-1) H^{T}(k)+V(k)\right)\right|^{1 / 2}} ;
$$

This is none other than the predictive distribution of the data at time $k$. Equation (21) can also be expressed in the more useful form:

$$
p\left(z(k) \mid Z^{k-1}, \mathcal{H}\right)=\frac{e^{-\phi(k) / 2}|P(k \mid k)|^{1 / 2}}{(|2 \pi V(k)||P(k \mid k-1)|)^{1 / 2}},
$$

where

$$
\phi(k)=z^{T}(k) V^{-1}(k) z(k)+\widehat{X}^{T}(k \mid k-1) P^{-1}(k \mid k-1) \widehat{X}(k \mid k-1)-\widehat{X}^{T}(k \mid k) P^{-1}(k \mid k) \widehat{X}(k \mid k) .
$$

The equivalence of (21) and (22) implies that the normalisers and exponents or each are identical, a fact that facilitates fast implementations. With appropriate modifications to the subscripts, these equations apply equally to data supplied from any subset of the independent sensors.

\subsection{The integrated likelihood of the discrete data}

The integrated likelihood of the discrete states $X_{D_{c(j)}}(k)$ given data $R_{c}^{k}$ is generated recursively, following equations (19) and (20), giving $p\left(R_{c}^{k} \mid \mathcal{H}\right)=\prod_{t=1}^{k} p\left(r_{c}(t) \mid R_{c}^{t-1}, \mathcal{H}\right)$. The recursive term corresponding to equation (21) can be evaluated directly,

$$
p\left(r_{c}(k) \mid R_{c}^{k-1}, \mathcal{H}\right)=\sum_{j=1}^{n_{c}} p\left(r_{c}(t) \mid X_{D_{c(j)}}\right) p\left(X_{D_{c(j)}} \mid R_{c}^{k-1} \cdot \mathcal{H}\right)
$$

\subsection{Track Fusion}

The distribution of the state of a fused track, given all the data from all the sensors, can be evaluated from the updates of the local track state distributions and the previous fused distribution. The relevant recursion is

$$
p\left(X(k) \mid Z_{a}^{k}\right)=K p\left(X(k) \mid Z_{a}^{k-1}\right) \prod_{s=1}^{S} \frac{p\left(X(k) \mid Z_{a}^{s, k}\right)}{p\left(X(k) \mid Z_{a}^{s, k-1}\right)},
$$


where $K$ is a normalising constant. This equation applies equally under both feedback and independent distributed tracking schemes (section 2) [10]. In the case of Gaussian kinematic tracks, this equation implies the following updates:

$$
\begin{aligned}
P^{-1}(k \mid k) & =P^{-1}(k \mid k-1)+\sum_{s=1}^{S} P_{s}^{-1}(k \mid k)-P_{s}^{-1}(k \mid k-1) \quad \text { and } \\
\widehat{X}(k \mid k) & =P(k \mid k)\left[\sum_{s=1}^{S} P_{s}^{-1}(k \mid k) \widehat{X}_{s}(k \mid k)-P_{s}^{-1}(k \mid k-1) \widehat{X}_{s}(k \mid k-1)\right] .
\end{aligned}
$$

The time updates are the same for all the trackers. The corresponding update for a discrete component $X_{D_{c}}$ follows directly from (25),

$$
p\left(X_{D_{c}}(k) \mid R^{k}\right)=K_{D_{c}} p\left(X_{D_{c}}(k) \mid R^{k-1}\right) \prod_{s=1}^{S} \frac{p\left(X_{D_{c}}(k) \mid R^{s, k}\right)}{p\left(X_{D_{c}}(k) \mid R^{s, k-1}\right)},
$$

where $K_{D_{c}}$ is the normaliser. If the measurement noise associated with the discrete process is correlated, no simple decomposition can be provided unless details are provided of the likelihood function of the data relevant to the discrete states.

\section{SINGLE-STAGE ASSOCIATION}

A naïve association mechanism is provided by the Bayes factor for testing the hypothesis that two tracks are formed by the same target $\left(\mathcal{H}_{0}\right)$ against the hypothesis that they are formed by two targets $\left(\mathcal{H}_{1}\right)$ using only the most recent measurements. This Bayes factor is

$$
\frac{P\left(\mathcal{H}_{0} \mid z_{a}^{1}(k), z_{a}^{2}(k)\right)}{P\left(\mathcal{H}_{1} \mid z_{a}^{1}(k), z_{a}^{2}(k)\right)}=\frac{P\left(\mathcal{H}_{0}\right) \int p\left(z_{a}^{1}(k), z_{a}^{2}(k) \mid X\right) p(X) d X}{P\left(\mathcal{H}_{1}\right) \int p\left(z_{a}^{1}(k) \mid X\right) p(X \mid s=1) d X \int p\left(z_{a}^{2}(k) \mid X\right) p(X \mid s=2) d X} .
$$

Unfortunately this approach is problematic because it is impossible to choose priors that do not have a strong influence. The prior distributions, $p(X), p(X \mid s=1)$ and $p(X \mid s=2)$, should be the same so as not to bias the result of the test, and they should be only weakly informative. However, such a selection would strongly favour $\mathcal{H}_{0}$. But it would also be unfair to choose them to be the posteriors generated by the previous stage at the central and local trackers; such priors would tend to be stronger than the likelihoods.

Bar-Shalom and Li [6, Chap. 8] took a different tack for deciding track association. They proposed a significance test of a $\chi^{2}$ statistic based on the difference between the local track state estimates under $\mathcal{H}_{0}$. If this state difference statistic becomes large, it is concluded that $\mathcal{H}_{1}$ is true. We summarise their approach below.

The means and covariance matrices of the states of the two tracks that are candidates for fusion are sent to the fusion centre by sensors $i$ and $j$. The target track states are $X_{i}(k)$ and $X_{j}(k)$; their respective estimates and covariance matrices are $\widehat{X}_{i}(k)$ and $\widehat{X}_{j}(k)$, and $P_{i}(k)$ and $P_{j}(k)$. The state difference statistic is

$$
\left[\widehat{X}_{i}(k)-\widehat{X}_{j}(k)\right]^{T} T_{i j}(k)^{-1}\left[\widehat{X}_{i}(k)-\widehat{X}_{j}(k)\right]
$$

and it is distributed as $\chi_{n}^{2}$ (chi-square with $n$ degrees of freedom), where $T_{i j}(k)=P_{i}(k \mid k)+P_{j}(k \mid k)-P_{i j}(k \mid k)-P_{j i}(k \mid k)$. The cross variances $P_{i j}(k \mid k)=\mathrm{E}\left\{\left[X_{i}(k)-\widehat{X}_{i}(k)\right]\left[X_{j}(k)-\widehat{X}_{j}(k)\right]^{T}\right\}=P_{j i}(k \mid k)^{T}$ can be evaluated recursively [6, p. 449] according to

$$
P_{i j}(k \mid k)=\left[I-K_{i}(k) H_{i}(k)\right]\left[F(k-1) P_{i j}(k-1 \mid k-1) F^{T}(k-1)+Q(k-1)\right]\left[I-K_{j}(k) H_{j}(k)\right]^{T},
$$

where $P_{i j}(0 \mid 0)$ is the zero matrix.

\section{MULTI-STAGE ASSOCIATION}

The problem with single stage methods is that they discount older data ${ }^{3}$. The drawbacks of the Bayes factor approach (28) are eliminated when we use all the data from the sections of the tracks to be fused. The data dominates, and the priors have very little effect.

The local state recursion (11) can be applied, under the assumption of conditional indepence, to give the fused state recursion

$$
p\left(X(k) \mid Z_{a}^{k}\right)=\frac{p\left(Z(k) \mid X_{G}(k)\right) p\left(X_{G}(k) \mid Z^{k-1}\right)}{\int p\left(Z(k) \mid X_{G}\right) p\left(X_{G} \mid Z^{k-1}\right) d X_{G}} \prod_{c=1}^{C} \frac{p\left(R_{c}(k) \mid X_{D_{c}}(k)\right) p\left(X_{D_{c}}(k) \mid R_{c}^{k-1}\right)}{\sum_{X_{D_{c}}=1}^{n_{c}} p\left(R_{c}(k) \mid X_{D_{c}}\right) p\left(X_{D_{c}} \mid R_{c}^{k-1}\right)}
$$

Since both local and fused recursions share the same independence structure, the Bayes factor for multistage association can be evaluated factor by factor:

$$
\frac{P\left(\mathcal{H}_{0} \mid Z_{a}^{k}\right)}{P\left(\mathcal{H}_{1} \mid Z_{a}^{k}\right)}=\frac{P\left(\mathcal{H}_{0}\right)}{P\left(\mathcal{H}_{1}\right)} \frac{P\left(Z^{k} \mid \mathcal{H}_{0}\right)}{P\left(Z^{k} \mid \mathcal{H}_{1}\right)} \prod_{c=1}^{C} \frac{P\left(R_{c}^{k} \mid \mathcal{H}_{0}\right)}{P\left(R_{c}^{k} \mid \mathcal{H}_{1}\right)}
$$

This expression can be generated evaluated recursively using equations (19) and (20) as follows,

$$
\frac{P\left(\mathcal{H}_{0} \mid Z_{a}^{k}\right)}{P\left(\mathcal{H}_{1} \mid Z_{a}^{k}\right)}=\frac{P\left(\mathcal{H}_{0} \mid Z_{a}^{k-1}\right)}{P\left(\mathcal{H}_{1} \mid Z_{a}^{k-1}\right)} \frac{P\left(Z(k) \mid Z^{k-1}, \mathcal{H}_{0}\right)}{P\left(Z(k) \mid Z^{k-1}, \mathcal{H}_{1}\right)} \prod_{c=1}^{C} \frac{P\left(r_{c}(k) \mid R_{c}^{k-1}, \mathcal{H}_{0}\right)}{P\left(r_{c}(k) \mid R_{c}^{k-1}, \mathcal{H}_{1}\right)}
$$

\footnotetext{
${ }^{3}$ Bar-Shalom and Li's statistic is sufficient for association using the entire track histories when $Q(t)=0 \quad \forall t: 0<t \leq k$.
} 
where the appropriate terms are substituted from equations (21) or (22), and (24).

A much better algorithm is available for Gaussian component than the simple application of (33). From the definitions of $V(k)$ (section 4.1) and $\phi(k)$ (equation 23), the terms in recursion (33) involving the data cancel. Only the state and covariance estimates need be retained for association (and for fusion - see section 4.4).

Depending on the engineering constraints, improvements may also come from using the normaliser of the first form (equation 21) in equation (22). Alternatively, a summation that allows a more compact recursion can be written by pairing terms between $\phi(k)$ and $\phi(k+1)$ :

$$
\begin{aligned}
\sum_{t=1}^{k} \phi(t) & =\sum_{t=1}^{k} Z^{T}(t) V(t) Z(t)+\sum_{t=1}^{k-1} \nu(t)+\Delta(k) \\
\text { where } \quad \Delta(k) & =\widehat{X}^{T}(1 \mid 0) P^{-1}(1 \mid 0) \widehat{X}(1 \mid 0)-\widehat{X}^{T}(k \mid k) P^{-1}(k \mid k) \widehat{X}(k \mid k), \\
\text { and } \quad \nu(t) & =\widehat{X}^{T}(t+1 \mid t) P^{-1}(t+1 \mid t) \widehat{X}(t+1 \mid t)-\widehat{X}^{T}(t \mid t) P^{-1}(t \mid t) \widehat{X}(t \mid t) \\
& =\widehat{X}^{T}(t \mid t) P^{-1}(t \mid t) F^{-1}(t) P(t+1 \mid t) F^{-T}(t) P^{-1}(t \mid t) \widehat{X}(t \mid t) .
\end{aligned}
$$

Having obtained real-time expressions for the association Bayes factor, we need a test to make a hard decision. The Bayesian approach to testing is somewhat different from the frequentist procedure, which decides the acceptance region for the null hypothesis by setting the probability of accidental rejection. The Bayesian view is that the only criterion for acceptance of a hypothesis is its posterior probability, but Bayesians prefer not to make hard decisions ${ }^{4}$. However, in this instance, if only for presentation to the operator, hard decisions are required, and the appropriate framework is minimum risk decision theory. This approach is detailed in [11].

\subsection{Associating two tracks}

These results are now applied to the problem of deciding whether to fuse two kinematic tracks. We compare the multistage Bayes factor method with Bar-Shalom and Li's single stage approach.

In this problem, the hypothesis $\mathcal{H}_{0}$ is that the targets observed by the two local trackers are identical, and the hypothesis $\mathcal{H}_{1}$ is that they are different. Taking logs, the recursion for the relevant Bayes factor is

$$
\log \left(\frac{P\left(\mathcal{H}_{0} \mid Z_{a}^{k}\right)}{P\left(\mathcal{H}_{1} \mid Z_{a}^{k}\right)}\right)=\frac{1}{2} \log (\Psi(k))-\frac{\Phi(k)}{2}+\log \left(\frac{P\left(\mathcal{H}_{0} \mid Z_{a}^{k-1}\right)}{P\left(\mathcal{H}_{1} \mid Z_{a}^{k-1}\right)}\right)
$$

where $\frac{P\left(\mathcal{H}_{0} \mid Z_{a}^{0}\right)}{P\left(\mathcal{H}_{1} \mid Z_{a}^{0}\right)}=\frac{P\left(\mathcal{H}_{0}\right)}{P\left(\mathcal{H}_{1}\right)}, \Phi(k)=\phi-\phi_{i}-\phi_{j}$ (see equation 23), and

$$
\Psi(k)=\left(\frac{\left|V_{i}(k)\right|\left|V_{j}(k)\right|}{|V(k)|} \frac{\left|P_{i}(k \mid k-1)\right|\left|P_{j}(k \mid k-1)\right|}{P_{i}(k \mid k)|| P_{j}(k \mid k) \mid} \frac{|P(k \mid k)|}{|P(k \mid k-1)|}\right) .
$$

It is interesting to compare the properties of the state difference statistic described in section 5 with this two-track kinematic Bayes factor. Figure 1 shows two targets on contrarotating linear spiral paths; their states coincide once every revolution. The state-difference test statistic clearly identifies them as different tracks when they are well separated, but it does not distinguish them when their states are similar ${ }^{5}$. The Bayes factor is an integrating measure, and it becomes steadily more strongly in favour of the two-target hypothesis.

The second simulation (figure 2) shows a single target under random walk, which is the model assumption built into the trackers. A test of $\chi_{4}^{2}$ at the $99.9 \%$ percentage point (18.47) would not reject the single track hypothesis. The log Bayes factor plot shows that the odds in favour of the single target hypothesis steadily mount. After a few iterations, the Bayes factor is conclusive.

The third simulation (figure 3) shows two targets on parallel spiral paths, one 3 units inside the other. It is hard to spot the difference between these two tracks by eye, but the state difference test statistic would trigger at the $99.9 \%$ percentage point quite frequently. The Bayes factor detects the difference conclusively.

\section{DISCUSSION}

The paper provides recursive forms for the Bayes factors for track fusion hypotheses under both Gaussian and discrete Markov dynamics. They enable track association and fusion processes to be run off-line or in real-time using either raw track data or saved statistics (means and covariances for Gaussian processes and state probabilities for Markov processes). Under strong independence assumptions they also apply to generalised tracks. They enable tracks to be associated on the basis of the data from the entire period of their coexistence.

Such closed form results are only available for models with decomposable independence graphs [12, 13], and this constraint eliminates all but a very few homogeneous time-series models [14]. However, approximate forms can be derived by using equation (33) directly (e.g. for IMM). The recursive methods described can be applied directly in MHT systems. Their property of using all the relevant data makes Bayes factors useful for discrimination, but it can cause problems by slowing down the discrimination of separating targets. A simple forgetting factor can be incorporated into the recursion (33) to alleviate this problem. The greatest practical difficulties in adopting these methods are imposed by the restrictions on the communication formats and the concealed, but necessary, "ad hockery" within proprietary trackers. Significant amounts of reworking may be necessary in full scale applications. Approximate forms of these results for nonlinear systems follow from linearising their target and measurement models. Utilisation of these methods would be helped by a thorough analysis of association hypothesis management, including, for example, issues such as gating, "negative" information and dropouts. An interesting issue arises from the use of Bayes factors: Bayesian tradition suggests that hypotheses should only be accepted on the basis of their posterior probability. However, in recursive applications, the conditions that motivate frequentist tests are present - the Bayes factor is a stochastic quantity and thresholds should be set cautiously; so a significance test may be appropriate.

\footnotetext{
${ }^{4}$ with some mathematical justification, however impractical

${ }^{5}$ It is an property of this motion that the difference between the states of the targets is periodic, and this accounts for the patterns of the state difference statistics and log Bayes factors.
} 

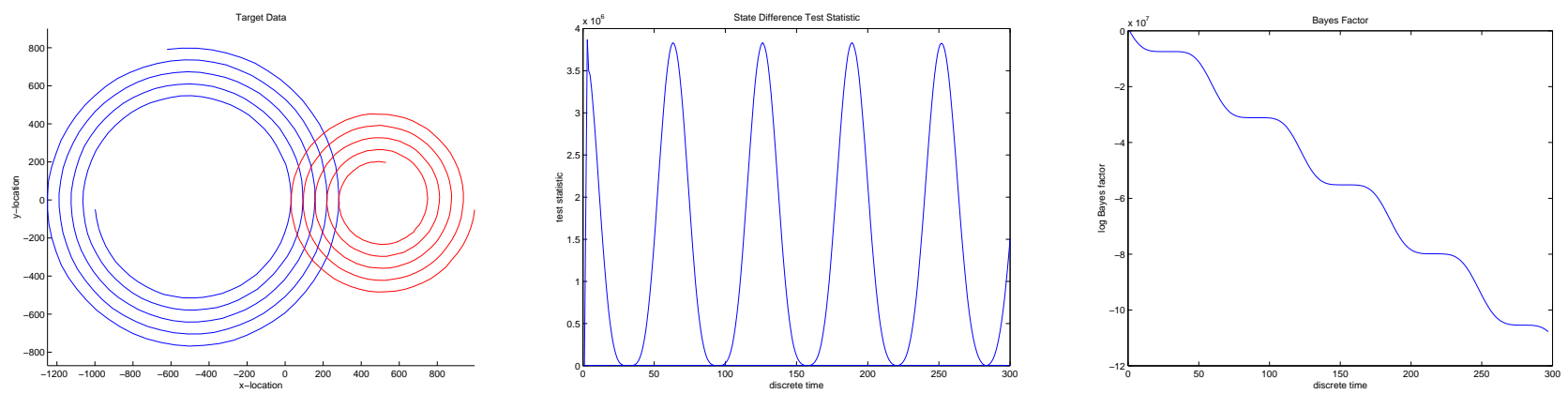

Figure 1: Two targets move on contrarotating spirals (left). The state-difference statistic detects misassociations when the target states differ (centre). The Bayes factor consistently shows that the targets are different (right).
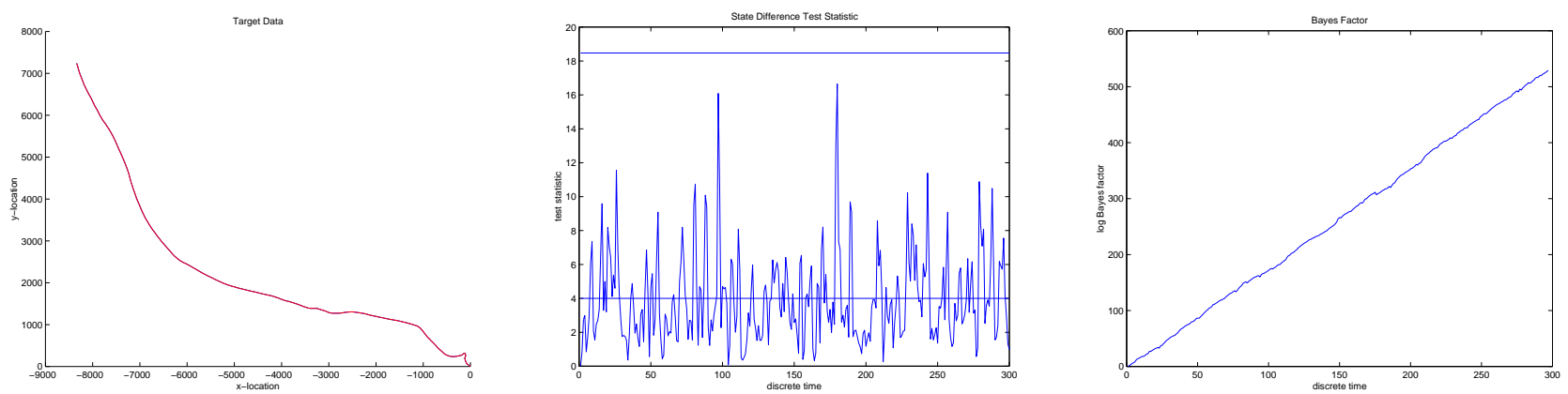

Figure 2: One target on a random walk is observed by two independent sensors (left). The state-difference statistic consistently favours the single-target hypothesis at the $99.9 \%$ level [18.47](centre). The Bayes factor is conclusive (right).
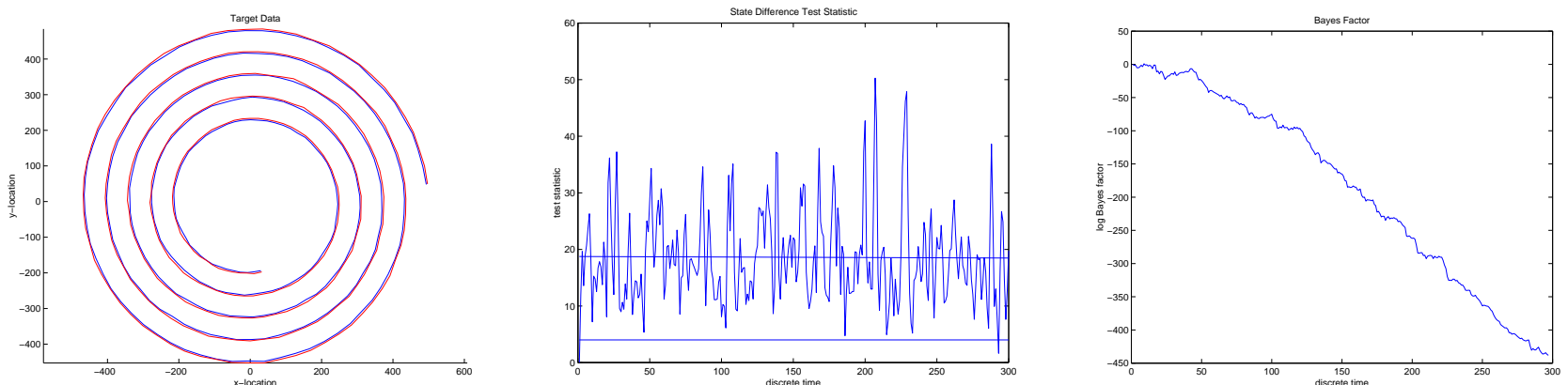

Figure 3: Two targets tracked from independent sensors on parallel spirals. The Bayes factor distinguishes the targets unambiguously (right), while the state-difference statistic cannot (centre).

\section{REFERENCES}

[1] R. Kass and A. Raftery, "Bayes factors,” J. Amer. Statistical Association, vol. 90, no. 430, pp. 773-795, 1995.

[2] F. Gustafsson, "The marginalized likelihood ratio test for detecting abrupt changes," IEEE Trans. Automatic Control, 1996.

[3] N. Okello, M. Roughan, and M. D., "Threat assessment using generalised tracking and Bayes nets," Tech. Rep. 1/97, Cooperative Research Centre for Sensor Signal and Information Processing, SPRI Building, Mawson Lakes, South Australia, 5095, 1997.

[4] N. Okello and D. McMichael, "Generalised tracking," in Proceedings, Information Decision and Control 99, (Adelaide), 1999.

[5] N. Okello, D. Tang, and D. McMichael, "Tracker: a simulator multisensor generalised tracking," in Proceedings, Information Decision and Control 99, (Adelaide), 1999.

[6] Y. Bar-Shalom and X.-R. Li, Multitarget-multisensor tracking: principles and techniques. YBS, 1995.

[7] K. Chang and Y. Bar-Shalom, "Distributed adaptive estimation with probabilistic data association," in 10th IFAC World Congress, (Munich, Germany), July 1987.

[8] B. D. O. Anderson and J. B. Moore, Optimal Filtering. New York: Prentice-Hall, 1979.

[9] S. B. Blackman, Multiple-target tracking with radar application. Norwood, MA: Artech House, 1986.

[10] C. Chong, S. Mori, and K. Chang, "Distributed multitarget multisensor tracking," in Multitarget-Multisensor Tracking: Applications and Advances (Y. Bar-Shalom, ed.), ch. 8, Artech House, 1990.

[11] M. Karan and D. McMichael, "Data fusion of submarine tactical data," Tech. Rep. 17/98, CSSIP, Adelaide, Australia, 1998.

[12] H.-G. Leimer, "Triangulated graphs with marked vertices," Annals of Discrete Mathematics, vol. 41, pp. 311-324, 1989.

[13] S. L. Lauritzen and N. Wermuth, "Graphical models for associations between variables, some of which are qualitative and some quantitative," Ann. Statist., vol. 17, pp. 31-57, 1989.

[14] M. I. Jordan, Z. Ghahramani, and T. S. Jaakkola, "An introduction to variational methods for graphical models," in Learning in Graphical Models (M. I. Jordan, ed.), pp. 105-161, Kulwer Academic Publishers, 1998. 